\title{
Chronic HCV treatment with peginterferon- ribavirin and severe tuberculosis re-activation
}

\author{
Roberto Manfredi*, Leonardo Calza \\ From $16^{\text {th }}$ International Symposium on HIV and Emerging Infectious Diseases \\ Marseille, France. 24-26 March 2010
}

\section{Background}

Tuberculosis $(\mathrm{T})$ may be reactivated following a primary, silent, and unknown $\mathrm{T}$ infection, when immunodeficiency (often jatrogenic in origin), or other risk factors (e.g. cancer, cachexia), become apparent. Post-primary $\mathrm{T}$ episodes were described also decades after a primary M. tuberculosis infection, in patients (p) who show apparently limited radiographic signs at chest X-ray. Some grade of immunodeficiency may also depend on the administration of associated IFN-ribavirin for an underlying chronic $\mathrm{HCV}$ hepatitis, as expressed by the frequent emerging of leuko-neutropenia, and altered cytokine network.

\section{Methods}

In a $\mathrm{p}$ aged $>50$ years with negative history of $\mathrm{T}$, an occasional chest X-ray showed fibrous-calcified infiltrates at upper right lobe. After 11 years, due to a progressive chronic HCV hepatitis, pegylated IFN-ribavirin were started for 7 months, until a sudden occurrence of cough-hemoptisis associated with a pulmonary lesion highly suggestive of $\mathrm{T}$ became apparent, in the same area where some reliquates of a primary $\mathrm{T}$ were demonstrated 11 years before.

\section{Results}

A HRCT examination pointed out 2 different excavated infiltrates. Both direct microscropy and culture of sputum-BAL proved positive for M. tuberculosis (susceptible to all tested compounds), while Mantoux-Quantiferon assays also tested positive. An absolute lymnphopenia (nadir 966 cells $/ \mu \mathrm{L}$ ), prompted a T-cell subset study, which showed an imbalance of the CD4/CD8 ratio (30/ $45 \%$ ), and an absolute CD4 count of 290 cells/ $\mu \mathrm{L}$. Notwithstanding 7 consecutive weeks of isoniazide, ethambutol, rifampicin and pyrazinamide administration, sputum examination remained positive, thus confirming the role of immunodeficiency is prompting a difficult-totreat $\mathrm{T}$. The adjunct of levofloxacin-amikacin-linezolid attained clinical-bacteriological cure, after 12 weeks.

\section{Discussion}

Waiting for human experimental data, two animal models demontrated that an increased release of immunosuppressive cytokines (IL-10-TGF- $\beta$ ), may prompt $T$ reactivation, while a maintained $T$-cell competence enhances $\mathrm{T}$ latency. Although a few cases of non-infectious lung involvement, interstitial pneumonia, and bronchiolitis obliterans were described during IFN therapy administered to transplant $\mathrm{p}$, reactivated $\mathrm{T}$ was excepional. The expected increase of therapeutic use of IFN and potent agents for the management of chronic hepatitis or other diseases, might support the reactivation of latent T. A careful medical history, Mantoux reaction, IGRA testing, and a chest X-ray, are mandatory before starting IFN therapy. In fact, the jatrogenic immunosuppression related to IFN-ribavirin may go beyond the expected leuko-lymphopenia, and also act against the quantitative-functional role of CD4 lymphocytes. This last circumstance may play a key role in $\mathrm{T}$ reactivation, when $\mathrm{T}$ latency is of concern.

Published: 11 May 2010

\section{doi:10.1186/1742-4690-7-S1-P83}

Cite this article as: Manfredi and Calza: Chronic HCV treatment with peginterferon-ribavirin and severe tuberculosis re-activation.

Retrovirology 2010 7(Suppl 1):P83.

* Correspondence: Roberto.manfredi@unibo.it

Infectious Diseases, University of Bologna, S. Orsola Hospital, Bologna, Italy 